\section{Cahiers de Narratologie}

Analyse et théorie narratives

17 | 2009

Stéréotype et narration littéraire

\title{
Le jeu entre stéréotypes et narration dans le roman noir
}

\section{Anissa Belhadjin}

\section{(2) OpenEdition}

\section{Journals}

Édition électronique

URL : http://journals.openedition.org/narratologie/1089

DOI : 10.4000/narratologie.1089

ISSN : $1765-307 X$

Éditeur

LIRCES

Référence électronique

Anissa Belhadjin, «Le jeu entre stéréotypes et narration dans le roman noir », Cahiers de Narratologie [En ligne], 17 | 2009, mis en ligne le 16 décembre 2009, consulté le 01 mai 2019. URL : http:// journals.openedition.org/narratologie/1089; DOI : 10.4000/narratologie.1089

Ce document a été généré automatiquement le 1 mai 2019.

\section{(c) $($ i) $(9)$}

Cahiers de Narratologie - Analyse et théorie narratives est mis à disposition selon les termes de la licence Creative Commons Attribution - Pas d'Utilisation Commerciale - Pas de Modification 4.0 International. 


\title{
Le jeu entre stéréotypes et narration dans le roman noir
}

\author{
Anissa Belhadjin
}

1 Le roman noir occupe une situation particulière dans l'aire de la littérature contemporaine. Ainsi, il fait partie intégrante du genre policier et est couramment considéré comme un avatar du roman-énigme classique, auquel ressortissent, par exemple, les aventures de Sherlock Holmes ou celles d'Hercule Poirot. Pourtant, ses origines sont plutôt à rechercher du côté des récits de pionniers américains, dont il émerge au début du XXe siècle. Et même si les détectives « hard boiled », premiers héros des romans noirs américains, menaient effectivement une enquête sur le modèle policier, celle-ci est loin d'être désormais constitutive du genre. Au point qu'à part le thème - très répandu dans la littérature - de la transgression criminelle, il est difficile d'isoler des critères définitoires pour le roman noir. Sans être tout à fait de la littérature policière dont il adopterait les structures et reprendrait les « topoï », le roman noir est pourtant considéré comme de la littérature de genre, considérée comme « un ensemble plus ou moins organisé de séquences stéréotypées ${ }^{1}{ }$. En effet, comment reconnaître un genre, comme le roman policier ou la science-fiction, si ce n'est par l'identification des stéréotypes qui le constituent? Ainsi, la récurrence de personnages extra-terrestres ou de voyages dans le temps suffit à classer une œuvre dans le genre de la science-fiction, tout comme un meurtre et un détective sont les conditions nécessaires du roman-énigme.

Identifier des stéréotypes dans un roman aboutit donc à le classer dans un genre, c'est-àdire dans le vaste domaine de la paralittérature, puisque les genres romanesques sont par définition paralittéraires pour la critique. Subsumé sous ce terme très négatif de " paralittérature », le genre n'est alors plus envisagé comme un espace singulier, mais au contraire assimilé au bloc compact des œuvres qui reproduisent des procédés, indépendamment de leur genre : ainsi, le modèle paralittéraire dégagé par Couégnas est-il constitué au premier chef par le recours régulier aux structures figées, par quoi se caractérise le stéréotype, par exemple «une tendance à la reprise inlassable des mêmes procédés[...], le recours systématique aux clichés [...], des personnages procédant d'une 
mimésis sommaire et réduits à des rôles allégoriques ${ }^{2} »$. Le stéréotype, tout comme la paralittérature, sont frappés au même coin, qui les bannit hors du domaine de la littérature.

Pourtant, le roman noir travaille constamment les modalités de son appartenance au genre en travaillant sur le stéréotype. Sur le genre il existe bien un genre noir, comme l'atteste l'emploi du terme "noir ", très vivace, malgré une essence par ailleurs difficile à saisir, en grande partie à cause des variations historiques des stéréotypes qui le constituent. Sur le stéréotype, le roman noir français contemporain lutte contre les stéréotypes thématiques - de l'inuentio, selon Dufays : personnages, décors, intrigue ${ }^{3}$ - par l'utilisation de structures narratives très particulières - la dispositio : «agencements [...] d'actions narratives ${ }^{4} »-$ si répandues aujourd'hui qu'elles sont probablement en train de créer un nouvel académisme, et partant, deviennent les nouveaux stéréotypes par quoi se définit le genre. Ce changement de pôle est bien caractéristique du mouvement continuel qui, depuis ses origines, assigne au genre noir de dépasser les frontières qui lui sont attribuées.

4 Comment, au sein de l'espace qui est le sien, dans le genre policier, au cœur de la paralittérature, le roman noir joue-t-il sur la notion de stéréotype, s'en nourrissant, le rejetant, en déplaçant les lignes de force, du stéréotype thématique au stéréotype narratif ? Quels sont les procédés particuliers qui sont employés, et que produisent-ils ?

5 La tradition universitaire considère implicitement que la « littérature de genre ", à quoi appartient le roman noir, s'oppose à celle de la «littérature », et, par extension, elle en acquiert une coloration dépréciative, comme sous la plume de Tzvetan Todorov :

Le chef-d'œuvre littéraire habituel, en un certain sens, n'entre dans aucun genre si ce n'est le sien propre; mais le chef-d'œuvre de la littérature de masses est précisément le livre qui s'inscrit le mieux dans son genre. Le roman policier a ses normes; faire « mieux » qu'elles ne le demandent, c'est en même temps faire moins bien ; qui veut "embellir" le roman policier, fait de la "littérature", non du roman policier. $^{5}$

S'établit ici une frontière nette entre « littérature » et « littérature de genre » : d'un côté, l'œuvre littéraire, originale et singulière; de l'autre, l'œuvre populaire qui pour être réussie doit se conformer aux stéréotypes de son genre d'appartenance. Pas question d'originalité, il s'agit "d'appliquer la recette», et la littérature de genre est alors dévaluée en raison de cette soumission aux stéréotypes. Cette opinion exprimée par Todorov, cohérente dans le contexte structuraliste, est encore largement partagée, même si le pas semble vite franchi entre « littérature de genre » et «sous-littérature », comme l'exprime encore cet autre jugement, selon quoi «seule la littérature de masse (histoires policières, romans-feuilletons, science-fiction, etc.) devrait appeler la notion de genre ; celle-ci serait inapplicable aux textes proprement littéraires ${ }^{6}{ }^{\prime}$.

7 En réservant le terme de "genre » à la production paralittéraire, qui « a ses normes », et en l'opposant à l'œuvre littéraire, seule à être originale, Todorov rabat assez nettement la question du stéréotype sur celle de la paralittérature. Or les œuvres topiques ou stéréotypiques ne sont évidemment pas l'apanage de la littérature de genre et, d'une manière générale, toute œuvre appartient à un genre, car « on ne saurait imaginer une œuvre littéraire qui se placerait dans une sorte de vide d'information et ne dépendrait

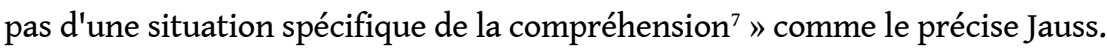


8 Pour Uri Eisenzweig, qui analyse la déconsidération dans laquelle est tenu le roman policier, la cause en est précisément « la nature spécifiquement générique de son objet », contractualisée par des stéréotypes, et pas l'œuvre en elle-même :

Ce n'est presque jamais en tant qu'œuvres individuelles, mais en tant qu'appartenant à un genre que [ces œuvres] sont marqués du seau de l'infamie paralittéraire. [...] Ce qui gêne, c'est le contrat. Le contrat, c'est-à-dire ce qui lie la forme au fond en promettant, par le biais d'un réseau de signes codés [...] une forme textuelle déterminée. ${ }^{8}$

9 Dans ce contexte, l'approche proposée par Jean-Marie Schaeffer offre des perspectives intéressantes pour l'analyse du lien entre stéréotype et généricité. Pour Schaeffer, l'appartenance au genre se manifeste aussi bien par la reprise incessante (la réduplication) que par l'écart (la transformation) aux textes qui servent de "modèle générique » et qui sont alors présents dans la nouvelle œuvre, tant du point de vue de la forme que de celui du thème ou de la visée. Ainsi, les chefs-d'œuvre évoqués par Todorov ne seraient pas en dehors des genres, mais s'y rattacheraient car ils « se qualifient non pas par une absence de traits génériques, mais au contraire par leur multiplicité extrême ${ }^{9}$ "; sans remettre en cause les stéréotypes, ces œuvres travaillent au contraire ceux-ci d'une manière particulière, et constituent donc un bon terrain d'étude. La généricité est alors fonction, non pas de la seule réduplication, mais aussi de la transformation, en fonction de «cette sorte de trame qui lie ensemble une classe textuelle et par rapport à laquelle le texte en question s'écrit : soit qu'il disparaisse à son tour dans la trame, soit qu'il la distorde et la démonte, mais toujours soit s'y intégrant, soit se l'intégrant ${ }^{10} »$. Cette conception très ouverte de la généricité permet de comprendre comment, au sein du genre noir, certains romans reproduisent inlassablement les mêmes stéréotypes alors que d'autres se signalent par leur inventivité, en « jouant » de ces stéréotypes.

10 Aujourd'hui, il est indéniable que de fortes contraintes génériques existent dans le roman noir, celles-là même qui le définissent. La première a à voir avec l'intrigue : le roman noir se caractérise par l'évocation d'une transgression criminelle des règles sociales. Mais de plus, l'absence de critère formel déplace les lignes de force du genre noir vers les critères thématiques. Ainsi, cette intrigue est saturée par les thèmes de la violence et de la mort et l'univers diégétique présente une vision négative de la société.

11 Cadré par cette intrigue et de ces thèmes, qui sont des stéréotypes génériques, le roman noir présente pourtant, historiquement, une grande diversité d'œuvres. Il y a loin, par exemple, entre l'enquête désabusée du roman "hard boiled» (voir les romans d'Hammett, à l'aube des années trente), les affrontements dans le « milieu » du roman de truands français (dans les années cinquante: Touchez pas au grisbi, ou Du rififi chez les hommes) ou les pamphlets gauchistes du néo-polar, dans les années soixante-dix. Le seul point commun entre tous : la criminalité, la violence. Aujourd'hui, le romancier noir contemporain, fortement ancré dans son temps, se veut souvent un témoin du malaise social. En ce sens, roman noir et roman-énigme (à la Agatha Christie) s'opposent : l'issue du roman-énigme célèbre le triomphe des conventions car l'enquêteur trouve le coupable, qui sera châtié, et l'ordre social revient. En revanche, dans le roman noir, même une fin heureuse ne préfigure en rien une société meilleure.

12 Ainsi, les thèmes s'entremêlent à l'intrigue pour donner sa particularité au roman noir, qui se réalise donc quand il présente une intrigue fondée sur un crime ou un délit, ayant un rapport avec l'expression par la violence d'un malaise social, ce qui entraîne une vision pessimiste, voire désespérée du monde. Une définition aussi lâche peut sembler 
circonscrire un genre précaire, pourtant la reconnaissance du genre est bien établie puisque l'expression «roman noir », qui désignait déjà le roman gothique, s'est imposée dans cette acception de « roman criminel social » lors de la création de la Série Noire au lendemain de la Deuxième Guerre mondiale en France, et le terme « noir ${ }^{11}$ » a essaimé aux Etats-Unis, ainsi que des expressions comme « film noir ${ }^{12}$ » ou même « roman noir $^{13}$ ». Leur emploi aujourd'hui, outre-Atlantique, indique assez qu'ils nomment là-bas un domaine qui n'était pas encore spécifié comme tel.

13 Le cadre générique du roman noir est donc formé de stéréotypes maillés en réseau, qui se situent sur le plan de l'inuentio. Très rigide, ce cadre est néanmoins travaillé aujourd'hui par le choix d'une narration particulière qui construit des romans hétérogènes, où la progression est discontinue ${ }^{14}$.

14 L'importance de l'intrigue comme critère constitutif du genre est probablement ce qui autorise cette hétérogénéité textuelle. Car si l'intrigue structure le genre, celui-ci, par voie de conséquence, définit les attentes du lecteur dans certaines limites et le jeu sur la narration a d'autant plus de place. Car quel que soit le morcellement du récit, le lecteur sait qu'il lit une histoire avec une intrigue spécifique. Pas un roman d'amour par exemple (donc une rencontre amoureuse ne saurait être le tout de l'intrigue) ou de science-fiction (si un personnage voit des soucoupes volantes, il y a une explication rationnelle: quelqu'un veut le faire passer pour fou)... ce qui oriente et délimite ses attentes. C'est ainsi que les stéréotypes, indubitablement contraignants, se trouvent inverser leurs effets supposés et offrir paradoxalement une bien plus grande liberté aux auteurs, en leur offrant de nouvelles perspectives.

15 Complexe est donc le rapport entre romans noirs et généricité, car le travail sur les stéréotypes est incessant, ce qui permet à Saint-Gelais d'affirmer «l'importance, au sein du domaine policier, du principe de variation, effervescent au point que la spécificité du genre policier est celle d'un espace en continuelle transformation ${ }^{15} »$. Aujourd'hui, cette effervescence s'applique à la narration: enfermés par les stéréotypes thématiques, les auteurs se saisissent de l'espace narratif pour contrebalancer les attentes du lecteur, comme nous allons le voir.

16 Est donc reconnu comme appartenant au genre noir le roman qui met en œuvre les stéréotypes ressortissant au dit genre. Ce qui apparait alors assez nettement au lecteur, c'est que le respect de cette contrainte thématique s'accompagne, le plus souvent, d'une grande hétérogénéité formelle, qui engendre dans la plupart des cas une véritable esthétique du discontinu. S'établit-il une sorte de compensation entre les différentes strates du récit, en l'occurrence celle de l'histoire et celle de la narration? Car en général, plus le récit est hétérogène, plus l'histoire elle-même use de stéréotypes : la plupart de ces romans noirs ne racontent jamais que des histoires de meurtres, de héros fatigués et de société corrompue. Le récit est donc le lieu d'une grande tension dans la mesure où les stéréotypes thématiques, qui comme tous les stéréotypes, privilégient la reconnaissance, entrent en collision avec les procédés narratifs, qui retardent la délivrance du sens : soit à cause du bouleversement de l'ordre temporel qui déstructure l'histoire, soit à cause d'un rapport particulier entre les différents points de vue, créant un ensemble polyphonique, soit par la composition d'ensemble du roman, faite de collages de toutes sortes.

17 Ces différents procédés dissociés ici pour les besoins de l'analyse peuvent être alliés et sont même très fréquemment associés dans le roman noir contemporain, au point qu'on en arrive aujourd'hui à une situation quasiment paradoxale. En effet, les auteurs travaillent sur la narration afin de compenser l'utilisation des thèmes stéréotypiques, 
véritables attendus, qui fondent le genre : ils visent donc à l'originalité - au sens aussi de renouveau. Mais toutes ces techniques narratives sont aujourd'hui si répandues qu'elles sont probablement en train de fonder un nouvel académisme, et deviennent à leur tour stéréotypiques, en participant de la définition du genre. Il s'agit donc ici de dresser une typologie de ces formes narratives, et d'observer les procédés qu'elles convoquent.

Selon Genette, les trois aspects particuliers liés au temps du récit sont l'ordre, la vitesse, la fréquence. L'ordre, qui est de ces différentes catégories celle qui est employée de la manière la plus significative dans le roman noir, est le rapport « entre l'ordre temporel de succession des événements dans la diégèse et l'ordre pseudo-temporel de leur disposition dans le récit ${ }^{16} »$. Distinguer temps de l'histoire et temps du récit permet, à l'instar des Formalistes Russes qui les premiers envisagèrent ce point précis, de poser de manière distincte la chronologie qui ressortit au temps diégétique - la fable - et la façon dont ces événements sont narrés - le sujet. Cela met alors en évidence qu'un récit parfaitement chronologique (qui respecte strictement l'ordre des événements) est illusoire, car des choix narratifs sont opérés, qui relèvent de la stratégie auctoriale. Dans les romans du genre noir, les "anachronies narratives ${ }^{17}$ " ont des enjeux très importants, se rapprochant en cela de la forme du roman-énigme, dite progressive/régressive, où avancer dans la lecture (le temps du récit) c'est remonter dans le temps (celui de la diégèse), ce qui révèle alors les circonstances du meurtre inaugural.

Dans le roman noir, peu d'histoires se déroulent donc linéairement, avec des événements narrés qui iraient vers une résolution présentée dans les dernières lignes. À la lecture tendue vers la conclusion succède une lecture morcelée, hétérogène, qui demande au lecteur une stratégie particulière : plutôt que de se laisser porter vers la fin du roman où sera résolue l'intrigue, il doit reconstituer les éléments du récit, dans une stratégie de dévoilement progressif qui n'est pas sans rappeler le code herméneutique en vigueur dans le roman-énigme. Cette récurrence du procédé de l'hétérogénéité temporelle dans le roman noir moderne pose essentiellement la question des enjeux: quelle peut être l'utilité d'un tel stéréotype narratif?

Les romans noirs qui emploient le décalage entre temps de l'histoire et temps du récit sont de deux sortes. Dans le premier cas, ce décalage est masqué ; des ellipses -implicites ou explicites - prennent alors place dans le récit, et seront comblées à la fin par d'ultimes analepses, sur le modèle suivant :

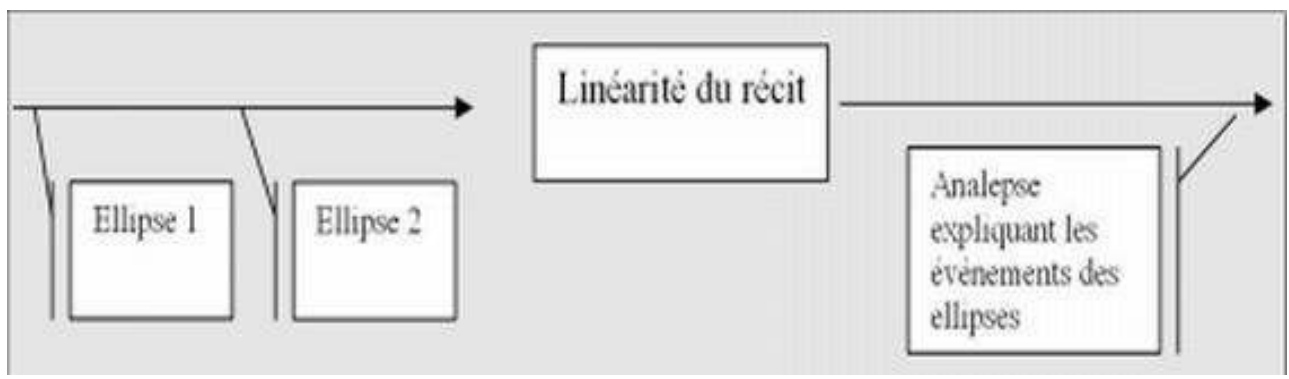

Parfois, ce décalage est exhibé et la succession des chapitres se fait clairement par l'alternance dans le récit de strates temporelles différentes, comme ci-dessous : 


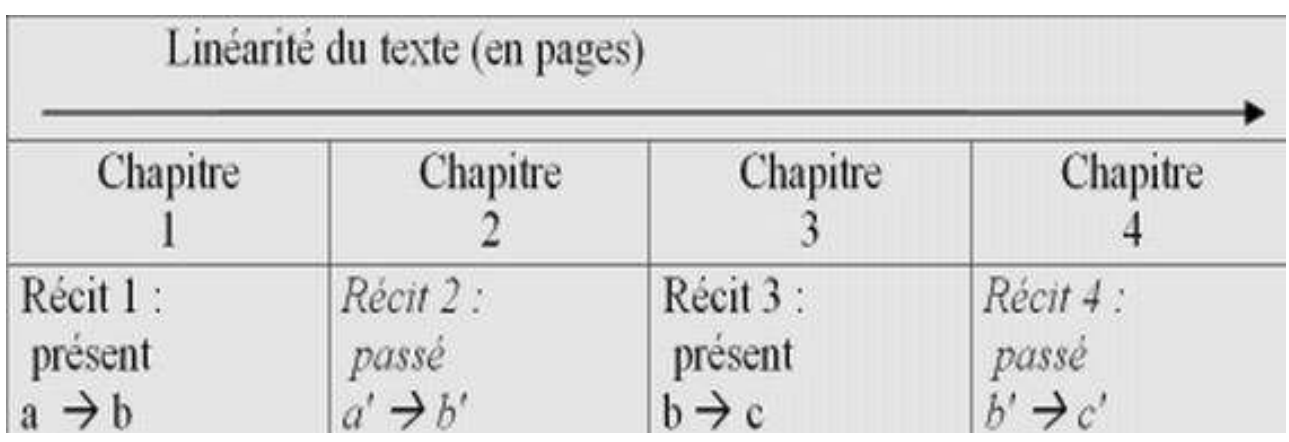

Dans les deux cas, le récit s'organise en général autour de l'absence d'une relation : un fait important est omis, qui ne sera révélé qu'à la toute fin du roman. Du coup, l'intrigue de ces romans, jusqu'aux dernières pages, est vraiment minimale, puisque les enjeux véritables sont masqués. Il y aurait donc là comme une capacité du lecteur de roman noir à supporter la banalité diégétique en supposant un enjeu herméneutique, même quand - et c'est le cas dans ces romans - il n'est dévoilé que dans l'excipit.

Ce principe du dévoilement tardif est ce qui fonde le roman-énigme. Du point de vue de la proximité générique institutionnelle, rien d'étonnant alors à ce que le lecteur de roman noir lise de cette façon particulière, savante, qui lui fait supposer des enjeux d'autant plus importants que la lecture du roman est banale. Mais dans le roman noir, le bouleversement temporel n'a plus la fonction explicative qu'il a dans le roman-énigme, où il est lié à la question "qui a tué ?»; sa fonction est plutôt de remettre en cause la logique du post hoc, ergo propter hoc. Au lecteur alors d'assembler les différents chapitres dans l'ordre de leur succession, afin que le récit prenne toute sa cohérence.

Ce terme de "multifocalisation» désigne, dans un récit, le changement de perspective, d'un personnage à un - ou plusieurs - autre(s). Il s'agit d'une technique narrative très courante dans le roman noir, tellement répandue qu'aujourd'hui, le lecteur averti est surpris si le roman qu'il lit n'est centré que sur un seul personnage. La multifocalisation n'est pas un procédé romanesque nouveau, puisqu'elle constitue le fondement de ce que l'on appelle le «roman unanimiste », et qu'emploie tout d'abord Jules Romains au début du XXe siècle. De Jules Romains à Dos Passos, de Dos Passos à James Ellroy, puis à la grande majorité des auteurs actuels, l'emploi de la multifocalisation s'inscrit d'ailleurs dans une filiation qui n'est pas inintéressante à observer.

Il existe de nombreuses variations dans l'emploi de la multifocalisation, aussi bien dans le nombre de perspectives utilisées pour construire le récit que dans la fréquence d'emploi du procédé lui-même (utilisé occasionnellement ou tout au long du récit): la multifocalisation autorise autant de variations que l'imagination peut en concevoir. En général pourtant, la majorité des romans utilisant cette forme de narration se limitent à une alternance de trois ou quatre points de vue - sinon il devient difficile de les homogénéiser pour reconstruire l'intrigue - tout au long du roman. Voici alors comment se présente, structurellement, un roman multifocalisé : 


\begin{tabular}{|c|c|c|}
\hline \multicolumn{3}{|c|}{ Linéarité du texte (en pages) } \\
\hline Chapitre 1 & Chapitre 2 & Chapitre 3 \\
\hline $\begin{array}{l}\text { Personnage } \mathrm{X} \\
\text { Lieu : A } \\
\text { Temps : } 1-2 \\
\text { Action } \alpha\end{array}$ & $\begin{array}{l}\text { Personnage } \mathrm{Y} \\
\text { Lieu : } \mathrm{B} \\
\text { Temps : } 1->2 \\
\text { Action : } \beta\end{array}$ & $\begin{array}{l}\text { Personnage } \mathrm{Z} \\
\text { Lieu : C } \\
\text { Temps : } 2->3 \\
\text { Raconte : } \chi\end{array}$ \\
\hline
\end{tabular}

Ce tableau synthétique souligne la collision entre la présentation matérielle du roman- ordonné, comme tout roman, d'une première à une dernière page dont les numéros se succèdent linéairement dans le texte - et sa composition globale. Celle-ci est fractionnée en divers chapitres qui possèdent leur logique endogène : ils sont centrés sur des personnages différents, dans des lieux différents, avec une temporalité qui leur est propre, et racontent des événements particuliers ; ce sont des mondes différents. Pas de passage ou de porosité d'un chapitre à l'autre, sinon incidemment, par le biais d'un lieu ou au hasard d'une action ou d'une évocation. Le lecteur, d'un chapitre à l'autre et d'un personnage focalisateur à l'autre, passe ainsi de monde en monde: les lieux, les personnages, voire les temporalités, peuvent être - et sont, le plus souvent - différents. Le roman tout entier est donc orchestré de manière polyphonique, la polyphonie étant entendue ici comme « un phénomène langagier d'essence esthétique, caractéristique de certains discours romanesques dans lesquels le narrateur fait parler des points de vue différents, sans paraître les subordonner au sien propre ${ }^{18}$ ».

Théoriquement, les romans polyphoniques présentent des mondes qui s'organisent de deux façons, selon qu'ils sont, entre eux, cloisonnés ou pas. Alors, d'un chapitre à l'autre et d'un personnage à l'autre, y a-t-il ou non une visibilité, quels sont les éléments communs? Les chapitres sont-ils étanches les uns par rapport aux autres, ou au contraire, les focalisateurs ne font-ils que présenter un point de vue différent sur une même histoire?

28 Dans la pratique, la ligne de partage n'est pas si nette, et la plupart des romans noirs sont mixtes, c'est-à-dire qu'en fonction des focalisateurs, la distance entre les mondes représentés peut être minime ou très importante: certains personnages ne se rencontreront jamais et d'autres très rapidement; leurs univers seront donc définitivement cloisonnés, ou non; pendant que d'autres personnages, dans d'autres mondes, seront mis en relation uniquement par le biais d'un indice commun... La distance est donc un critère variable et fluctue au cours du récit. De plus, une autre "distance » intervient ici, celle qui fonde l'opposition entre mimésis et diégésis, en fonction de quoi les mondes représentés peuvent être mis en rapport soit de façon directe, soit indirectement par le récit d'un personnage.

29 Dans le roman noir, les enjeux de la multifocalisation sont finalement assez éloignés de la construction unanimiste. En effet, une intrigue multifocalisée n'est pas forcément liée à la représentation d'une fresque de grande ampleur, autrement dit, la multifocalisation n'est pas que la somme de points de vue individuels pour construire un point de vue global. Dans la plupart des romans noirs, la multifocalisation sert plus ou moins à construire une intrigue, non pas seulement « à partir » des perspectives, mais « entre » celles-ci. Au-delà d'un procédé qui permet l'expression du point de vue de plusieurs personnages, la 
multifocalisation est donc un moyen de jouer avec les interstices laissés entre les différentes perspectives.

30 La multifocalisation, qui consiste habituellement à faire varier les perspectives d'un personnage de l'histoire à un - ou plusieurs - autre(s) peut être utilisée non pas seulement pour raconter le récit selon tel ou tel point de vue, mais aussi pour présenter des formes de discours très différentes, ressortissant au discours romanesque certes, mais aussi par exemple au dialogue, au journal intime, à la poésie ou à la dépêche de presse. Le roman n'est donc pas seulement la progression d'un récit en fonction de focalisateurs alternés, mais utilise aussi ce que l'on pourrait appeler un "collage ${ }^{19}$ " plutôt qu'un montage, de discours au service d'une intrigue somme toute assez topique. Or, la plupart de ces collages ont ceci de particulier qu'ils imitent des discours non-fictionnels ou nonromanesques. Comment les diverses formes de discours sont-elles au service de la forme romanesque?

31 Une remarque tout d'abord à propos du critère de la « forme » romanesque : celle-ci, dans le cadre de la littérature de fiction (celle que Gérard Genette oppose, dans Fiction et diction, à la littérature de diction, déterminée précisément par ses caractéristiques formelles) n'est pas un critère constitutif, contrairement au « caractère imaginaire de ses objets ", critère qui, lui, se situe plutôt du côté du contenu. L'appréciation de l'appartenance, ou non, d'un roman à la littérature tient, comme le rappelle Genette, à sa littérarité, qui se rapporte à son écriture ${ }^{20}$ ou à son thème (" critère thématique ») mais pas à sa forme. Il n'y a pas, en effet, de forme spécifique au texte romanesque, qui peut feindre de n'être pas fictionnel: une fausse biographie, quel que soit son degré de ressemblance avec une biographie authentique, est imaginaire et donc fictionnelle. C'est ce que Michal Glowinski appelle la « mimésis formelle », dont il donne cette définition :

C'est une imitation, par le moyen d'une forme donnée, d'autres modes de discours littéraires, paralittéraires et extralittéraires, ainsi que, selon un procédé relativement commun, du langage ordinaire. [...] Nous avons donc affaire à un certain type de stylisation. C'est la raison pour laquelle on ne peut parler de mimésis formelle que lorsque se manifeste une certaine tension, un certain jeu entre différents modes d'expression; par exemple, lorsqu'un roman fait usage des règles structurelles qui appartiennent au Journal intime. [...] C'est cette tension qui constitue le critère fondamental de la mimésis formelle ${ }^{21}$.

32 Mais, toujours selon Glowinski, l'imitation ne signifie pas pour autant que le récit reproduise totalement les règles de la forme qu'il imite. Il y a nécessairement distorsion entre les deux, et « collision violente de règles hétérogènes ${ }^{22}$ » ne serait-ce que parce qu'il s'opère une fictionnalisation de la structure imitée : la visée en est donc différente.

33 Dans beaucoup de romans noirs, c'est ce principe de mimésis formelle qui gouverne la progression, engendrant une hétérogénéité très marquée. En effet, bien qu'étant totalement fictionnels, ces romans sont fondés sur l'alternance de différents chapitres dont certains adoptent une forme ni romanesque, ni même fictionnelle. C'est le cas par exemple dans French Tabloïds, roman noir de Jean-Hugues Oppel dont certains passages imitent des courriels (jusque dans la reproduction de l'adresse) ou sont une compilation de véritables titres de unes de journaux quotidiens. Dans ce cadre précis, il faut supposer que cette hétérogénéité se trouve finalement réduite, puisque la question de la fictionnalité et du romanesque ne se pose pas, et que ces formes discursives multiples, et variées, sont malgré tout cohérentes.

34 Ce qui permet précisément de différencier la fiction de la non-fiction est le caractère imaginaire, qui est le propre du texte de fiction. Dans un récit non-fictionnel, les faits 
narrés préexistent au récit et sont vérifiables, alors que la fiction invente les faits en les racontant. Un texte de fiction doit également s'inscrire dans un cadre pragmatique bien particulier, que Jean-Marie Schaeffer définit comme celui de la feintise ludique partagée, sans quoi la fiction serait au mieux un malentendu, au pire un mensonge. Mais il y a plus : en tant que fiction " romanesque », ils doivent présenter un autre critère qui a trait à la médiation du narrateur.

Or, de nombreux chapitres peuvent ne pas avoir cette caractéristique particulière de présenter une médiation narratoriale. Ils sont construits selon le principe de «mimésis formelle», et donc se présentent comme s'ils n'étaient pas de la fiction romanesque. Rappelons en effet qu'« il n'y a pas de mimésis formelle lorsqu'un récit donné se borne à manifester les structures inhérentes au genre même ou à la convention stylistique dont il relève $^{23}$ ». Ces chapitres romanesques peuvent ressortir donc au dialogue, au poème, au texte de presse ou au journal intime... tous genres non-romanesques, ou non-fictionnels, ou même non-littéraires. Ce qui complique ici la description de ces différents discours, c'est la relation d'inclusion et d'opposition dans laquelle ils se trouvent les uns par rapport aux autres. Ainsi, le roman fait partie de la catégorie plus générale de la fiction (qui comprend aussi, par exemple, les genres fictionnels non-romanesques comme le théâtre), elle-même comprise dans celle de la littérature (dans laquelle se trouve aussi la non-fiction: la poésie, ou l'essai, ou l'autobiographie), qui s'oppose à l'usage nonlittéraire de l'écrit (comme l'article de journal, ou la lettre, ou le journal intime, ou le mode d'emploi...). De plus, certaines de ces catégories sont fluctuantes, en fonction des critères de la « poétique conditionaliste ${ }^{24}$ » définie par Genette.

Les collages employés dans les romans noirs ont donc pour effet de mêler des discours très différents et, en quelque sorte, neutralisent l'emploi des stéréotypes thématiques en les diluant dans ces discours très hétérogènes.

37 Anachronies narratives, multifocalisation ou collages: tels sont dans le roman noir contemporain les stéréotypes narratifs, en ce sens qu'on les observe dans la plus grande partie de la production française actuelle. Leur lien affirmé avec le genre noir peut s'expliquer par les compétences du lecteur de roman noir, lequel acquiert une compétence particulière qui lui permet de s'adapter à ces formes hétérogènes et de reconnaître une intrigue noire grâce à - et non pas « malgré » - ces procédés narratifs qui sont comme un nouveau stéréotype générique. Toutes ces techniques narratives ont pour effet l'éclatement la narration. Cet éclatement peut donc se réaliser de plusieurs façons, soit que l'auteur distorde le temps de l'histoire, ce qui fait naître un récit où les analepses ont une fonction déterminante dans la construction du sens, soit qu'il multiplie les points de vue, l'intrigue se trouvant alors diffractée en une multitude de faisceaux qui ne convergent pas obligatoirement, soit en collant des extraits non romanesques et en jouant sur la mimésis formelle. Dans ces différents cas, c'est au lecteur que revient la charge d'assembler le tout en extrayant un sens, une intrigue, une sorte de dénominateur commun.

38 C'est donc là le fonctionnement commun à tous ces procédés que nous avons analysés, qui appellent la participation active du lecteur, dans le mesure où le récit ne se borne pas à l'addition de ses chapitres, mais nécessite également des compétences particulières, liées à l'effort que demande la reconstruction de l'histoire dans sa linéarité, seule condition à laquelle l'intrigue peut être comprise.

Dans le roman noir, l'utilisation des procédés narratifs dont nous venons de rendre compte a pour effet de créer une grande hétérogénéité; quels peuvent en être les 
enjeux ? Pour paraphraser Borges selon qui « le roman policier a créé un type spécial de lecteurs ${ }^{25} »$, quels lecteurs le roman noir a-t-il créé ?

40 A partir de ce que Dufays appelle le "précadrage typo-générique ${ }^{26}$ ", qui se fait à partir des seuils de l'œuvre (nom de l'auteur, édition, collection de publication, illustration...), le lecteur de roman noir «sait » qu'il lit un récit à intrigue, appartenant à un genre cadré par des stéréotypes thématiques. Ce « savoir » crée une attente particulière, car le genre programme sa lecture. Dans la «lecture noire", tout fonctionne donc, du côté de la réception, comme si une sorte de grille de lecture structurait les attentes du lecteur. Non pas que l'on veuille revenir ici aux conceptions de la structure immanente du texte, chère à la poétique structuraliste. Mais en accord avec Saint-Gelais, selon qui « les processus de lecture, loin de présenter un caractère homogène, sont génériquement différenciés, de sorte qu'on puisse parler de [...] lecture policière ${ }^{27} "$, on peut penser que certains indices $\mathrm{du}$ texte enclenchent ce processus. Tout d'abord, au niveau de la diégèse, le thème de la transgression des règles sociales (vol, agression, meurtre...) est déterminant puisqu'il a le statut de stéréotype et permet de combler une attente générique. Ensuite, pour la narration, la multifocalisation, étant donné sa fréquence d'emploi, est en même temps un signe où le lecteur est invité à reconnaître la structuration d'une intrigue, à travers une question qui porte sur la mise en commun de tous ces mondes présentés et l'indice d'un conflit puisqu'en général, ces mondes s'opposent. Si la multifocalisation ne structure pas le récit, il sera lacunaire et incomplet, jouant sur les anachronies narratives ou les collages. Le manque de sens, d'où naît l'intrigue, est engendré par ces choix particuliers.

41 Postulons donc que les stéréotypes thématiques, qui définissent le genre, rendent nécessaire l'emploi de ces procédés narratifs qui en contrebalancent l'effet; même si la fréquence de leur emploi, leur structure figée, versent à leur tour ceux-ci au rang des procédés stéréotypiques, tant il est vrai que le " type spécial de lecteur » évoqué ci-dessus lit chaque roman d'un genre à la lumière de tous les autres. Parallèlement à cet emploi " compensatoire " pourrait-on dire, ces mêmes procédés sont indissociables d'un jeu, comme on peut appeler cette relation dialogique qui se tisse entre auteur et lecteur de romans noirs. Ce sont ces deux enjeux fondamentaux qu'il s'agit d'envisager.

Les romans noirs s'inscrivent dans une architextualitée ${ }^{28}$, celle du genre noir, et cela n'est évidemment pas sans conséquence sur leur production, ni sur leur lecture, puisque «la perception générique, on le sait, oriente et détermine dans une large mesure «l'horizon d'attente » du lecteur, et donc la réception de l'œuvre ${ }^{29}$ ». Le genre implique donc un dialogisme spécifique, puisque les attentes du lecteur étant en même temps configurées par le genre et bornées par ses limites, l'auteur joue donc sur ces attentes et ces limites.

43 S'inscrivant d'emblée dans un genre, la « lecture noire » détermine donc la position du lecteur par rapport au roman qu'il lit. Le lecteur sait que l'intrigue implique une certaine tension, qui agit à deux niveaux au moins : « dans » le déroulement de l'histoire - que vat-il se passer ? - mais aussi « en dehors » de cette histoire, dans la façon dont elle va être racontée.

44 Dans le cadre de nos romans, récits à intrigue, la relation dialogique joue donc entre l'auteur et le lecteur - via le texte littéraire - d'une manière tout à fait particulière. En effet, il ne s'agit pas tant d'expliciter un sens prédéfini de manière à lever toutes les ambiguïtés que de faire naître de multiples interprétations en multipliant les sens. Ce n'est alors que lorsque le récit s'achève qu'il est possible de privilégier l'une de ces interprétations, plutôt qu'une autre. Aussi les thèmes, comme l'intrigue, en même temps qu'ils sont des attendus génériques, doivent-ils être, autant que possible, disséminés au fil 
du texte; pour ce faire, l'intrigue doit être fractionnée, maintenue dans les bornes des différents mondes explorés par la diégèse. Ainsi, la multifocalisation rend imperméables les uns aux autres les mondes où agissent chacun des personnages focalisateurs; le jeu sur le temps du récit a comme effet de disloquer la logique des actions qui ne se succèdent plus, tout comme les collages, qui exhibent l'hétérogénéité du récit, en attirant l'attention du lecteur sur la nécessité de la recomposition.

Le genre noir impose à l'auteur d'aller toujours plus loin dans le procédé de la tension narrative. En effet, le lecteur de romans noirs intègre à son "catalogue des leurres » les pièges auxquels il a déjà été pris dans ses lectures ; en lisant un autre roman, si l'intrigue présente des indices similaires à ceux de lectures antérieures, il en anticipera la résolution. Et comme les surprises portant sur le contenu diégétique ne sont pas infinies, s'ajoutant toujours à la compétence encyclopédique du lecteur - à son catalogue des leurres - il peut sembler logique de remettre en cause la narration elle-même... L'emploi de ces procédés narratifs qui disséminent le sens de l'histoire est donc une conséquence des règles génériques qui reposent sur les stéréotypes de l'inuentio.

46 Étant donné l'importance de ces stéréotypes narratifs qui jouent sur les attentes du lecteur de romans noirs, on peut imaginer que les romans de ce genre, autant que de raconter une histoire, illustrent la relation entre auteur et lecteur : volonté de surprendre d'un côté, recherche des meilleurs moyens pour cela; attente d'être surpris de l'autre, anticipation de l'intrigue et de ses effets. Il se crée donc une tension entre les stéréotypes ressortissant à l'histoire, qui cadrent le genre, et l'acte narratif qui a pour effet de les masquer, de les rendre moins lisibles. Cette remise en cause, participant du genre, devient alors stéréotype à son tour. C'est donc une relation dialogique particulière qui se tisse au travers du texte, qui impose de s'interroger sur la part de « jeu » qu'elle comporte nécessairement, ce que nous allons faire à présent.

C'est donc cette voie du roman noir comme jeu que nous aimerions explorer à présent, un jeu qui est dans les « enjeux» de la lecture. Il s'agit de recomposer, de reconstruire un texte hétérogène, un texte qui a du « jeu », où les manques sont plus importants, du point de vue stratégique, que la prolifère et stéréotypique diégèse, et qui constitue l'aire du jeu.

48 L'entrée en jeu dans le roman noir commence, pour une large part, dès les seuils $d$ l'œuvre, et notamment le "prière d'insérer", qui souvent se présente comme un véritable défi au lecteur ${ }^{30}$. Mais l'espace du jeu, c'est à proprement parler le roman luimême. Nos romans, qui sont fondamentalement hétérogènes, présentent donc des indéterminations à la lecture. Il est très fructueux de les analyser comme les «blancs » qu'Iser définit comme "l'occupation de certains lieux du système textuel par les représentations du lecteur [...]. Dans la mesure où les blancs signalent l'omission d'une relation, ils permettent au lecteur de se la représenter librement et "disparaissent" aussitôt qu'elle est établie ${ }^{31} »$. Pour Iser, les blancs (littéralement : Leerstellen, places vides) font naître chez le lecteur une attente, et participent d'une esthétique particulière propre au roman moderne.

Lorsqu'il lit un roman noir, qui se présente de manière aussi hétérogène qu'un texte moderne, le lecteur sait qu'un sens le sous-tend et que s'il n'est pas à même de le trouver, il lui sera finalement apporté. Cette attente inhérente au genre peut bien sûr être contournée, car certains romans refusent cette détermination finale. Il n'empêche qu'elle structure le genre assez fortement, et constitue une part importante du jeu. 
50 Cette détermination finale du sens, preuve de «ce positivisme policier, cette euphorie herméneutique, cette garantie illusoire de complétude sémiologique, et idéologique ${ }^{32}$ " est donc la marque du genre. Le texte policier engendre l'attente pour finalement la résoudre; il y a donc jeu sur cette attente dont le lecteur sait qu'elle va être comblée, contrairement au roman moderne évoqué par Iser où ce « jeu d'attente [...] a montré que l'idée d'une continuité, évoquée par le thème et dès lors attendue par le lecteur devait être abandonnée par le lecteur lui-même ${ }^{33}$ ». Le roman noir est lié fortement à la tension narrative $e^{34}$, qui engendre curiosité et suspense, par quoi son auteur déjoue la prévisibilité de son histoire.

51 Par exemple, tous les romans multifocalisés- avec ou sans analepses - jouent sur l'attente du lecteur, qui attend la mise en relation des différents mondes diégétiques. Même chose pour les romans qui jouent sur le temps du récit, puisque les analepses influent sur la progression de l'histoire racontée et la résolution de l'intrigue. À cet égard, il y a donc une collision assez nette entre l'écriture, qui vise à mimer la densité, la rapidité des actions, et la progression narrative, qui joue sur le retardement. Toutes deux étant évidemment liées puisque le lecteur est entraîné par le rythme des actions alors que la structure de l'œuvre est profondément dilatoire. Lié au processus herméneutique, le jeu est donc manifeste pour ces romans qui portent la tension narrative à son comble, ces textes où « ça joue » dans l'interprétation, entre l'œuvre et le lecteur, et où la part du jeu est essentiellement engendrée par la différence entre linéarité de la lecture et fractionnement narratif.

52 Le jeu est donc principalement circonscrit par les blancs du texte qui, en tant qu'ils déterminent et structurent les attentes du lecteur, ont un rôle fondamental. Même si, contrairement au roman moderne dont parle Iser, ces blancs ne sont pas tant au service d'une esthétique particulière qu'au service d'un jeu, exacerbant l'attente du lecteur pour mieux pouvoir la résoudre. Les blancs sont en rapport direct avec l'espace et le temps romanesques, ludiques par essence dans le roman noir, puisqu'ils sont à recomposer par le lecteur. Le résultat est que, comme le dit Saint-Gelais, « un roman policier est moins un "objet" qu'un "dispositif" dont le fonctionnement passe par l'intervention active du lecteur ${ }^{35} »$.

Dans le roman noir, les auteurs sont nombreux à se réclamer de la littérature populaire, en droite ligne du XIXe siècle : souffle épique, grandes fresques, suspense leur paraissent fondamentaux dans le genre romanesque. Ces auteurs ont donc tendance à rejeter massivement la littérature blanche, qualifiée par eux de littérature d'introspection, d'autant que les mêmes qui célèbrent le roman a-générique-critiques littéraires, institution universitaire - sont ceux qui nient l'intérêt de la littérature de genre. Un des facteurs de cohésion du groupe, composé d'auteurs qui fondent leur identité d'écrivains de romans noirs en s'opposant à la littérature blanche, a-générique, se trouve donc dans la particularité qu'ils s'attribuent : raconter des histoires. Comme, d'autre part, le genre noir impose une cohérence thématique assez forte, celle-ci, en même temps qu'elle est fondée sur des stéréotypes vivaces, offre pourtant la possibilité d'une structure très libre. On peut alors penser qu'il en est pour la contrainte thématique comme pour l'image du genre: de même que les auteurs renversent les valeurs attribuées à leur genre qu'ils considèrent comme plus fécond que les autres, ils transforment les contraintes thématiques en atout et s'en saisissent pour faire de leurs romans des œuvres novatrices. D'où le travail sur la narration, qui, en même temps qu'il permet de compenser l'emploi d'intrigues et de thèmes très codés (meurtres, critique sociale, dénonciation des 
problèmes de notre temps par un héros en marge de la société, faisant figure d'antihéros) permet de les disséminer au fil du texte en une multitude d'univers qui semblent indépendants les uns des autres jusqu'à ce que le lecteur recompose une intrigue unique qui opère la "synthèse de l'hétérogène ${ }^{36}$ »; cette expression de Ricœur est tout à fait indiquée pour beaucoup de romans noirs qui présentent une collision de mondes fictionnels sans aucune homogénéité reliés par une intrigue, dans des textes où "ça joue », et où le « jeu " est à prendre dans les deux sens de ce terme : le jeu, ludique, et le jeu, spatial, qui désunit les deux parties d'un tout. Certes, certains romans noirs aujourd'hui, se rapprochant en cela du roman policier postmoderne défini par Stefano Tani, "antiroman policier " présentent des fins très ambiguës et deviennent «le contraire exact d'un roman policier, qui trompe son monde en ne lui servant pas à point nommé la solution toute faite qu'il attend ${ }^{37}$ ». C'est le cas, par exemple, des romans de Claude Amoz. Mais de tels romans, où la fin ne permet pas de réduire l'hétérogénéité, sont aussi fondés sur le principe du jeu en soulignant à quel point le lecteur est prisonnier de l'intrigue dont il attend la résolution. Plus généralement, l'emploi de ces procédés narratifs, très courant dans le roman noir contemporain, devient stéréotypique à son tour et est désormais quasiment indissociable du genre.

\section{BIBLIOGRAPHIE}

BARONI Raphaël, «Tension narrative, curiosité et suspense : les deux niveaux de la séquence narrative», www.vox-poetica.com

BORGES Jorge Luis, Conférences, [Paris :] Gallimard, 1985.

COUÉGNAS Daniel, Introduction à la paralittérature, Paris : Seuil, 1992.

DUFAYS Jean-Louis, Stéréotype et lecture : essai sur la réception littéraire, Liège : Mardaga, 1994.

DUFAYS Jean-Louis, « Le Stéréotype, un concept-clé pour lire, penser et enseigner la littérature » in Marges Linguistiques, mars 2001.

EISENZWEIG Uri, « Présentation du genre » in Littérature, n49, février 1983.

GENETTE Gérard, Figures III, Paris : Seuil, 1972.

GENETTE Gérard, Palimpsestes : la littérature au second degré, Paris : Seuil, 1982.

GENETTE Gérard, Fiction et diction, Paris : Seuil, 1991.

GLOWINSKI Michal, «Sur le roman à la première personne » in Poétique, n², novembre 1987, p. 497-507.

ISER Wolfgang, L'Acte de lecture : théorie de l'effet esthétique, Bruxelles : Mardaga, 1985.

JAUSS Hans Robert, «Littérature médiévale et théorie des genres » in Théorie des genres/ Gérard Genette, Tzvetan Todorov (s. la dir.), Paris : Seuil, 1986.

MELLIER Denis, MENEGALDo, Gilles (s. la dir.), Formes policières du roman contemporain, La Licorne, $\mathrm{n}^{\circ}$ 44, 1998. 
RABATEL Alain, « La dialogisation au cœur du couple polyphonie/ dialogisme chez Bakhtine » in Revue romane, $\mathrm{n}^{\circ} 41-1$, avril 2006, p. 55-80.

REUTER Yves, Le Roman policier, Paris : Nathan, 1997.

RICEUR Paul, Temps et récit I : L'Intrigue et le récit historique, Paris : Seuil, 1983.

SCHAEFFER Jean-Marie, « Du texte au genre » in Théorie des genres/ Gérard Genette, Tzvetan

Todorov (s. la dir.), Paris : Seuil, 1986.

SAINT-GELAIS Richard, « Rudiments de lecture policière » in Revue belge de philologie et d'histoire, $\mathrm{n}^{\circ}$ $75,1997$.

TANI Stefano, « Le détective écartelé » in Diogène n¹20, 1982.

TODOROV Tzvetan, « Typologie du roman policier » in Poétique de la prose, Paris : Seuil, 1980, p. 9-19.

TODOROV Tzvetan, Introduction à la littérature fantastique, Paris : Seuil, 1970.

\section{NOTES}

1. Jean-Louis Dufays, Stéréotype et lecture, Mardaga, 1994, p. 92.

2. Daniel Couégnas, Introduction à la paralittérature, Seuil, 1992.

3. Jean-Louis Dufays, Stéréotype et lecture, op. cit. p. 93-96.

4.Jean-Louis Dufays, «Le Stéréotype, un concept-clé pour lire, penser et enseigner la littérature ", in Marges Linguistiques, mars 2001.

5. Tzvetan Todorov, « Typologie du roman policier », in Poétique de la prose, Seuil, 1971, p. 10.

6. Tzvetan Todorov, Introduction à la littérature fantastique, Seuil, 1970, p. 10-11.

7. Hans Robert Jauss, « Littérature médiévale et théorie des genres » (1970) in Théorie des genres, Seuil, 1986, p. 42.

8. Uri Eisenzweig, « Présentation du genre » in Littérature, n49, février 1983.

9. Jean-Marie Schaeffer, « Du texte au genre » (1983) in Théorie des genres, Seuil, 1986, p. 203.

10. Ibid. p. 204.

11.Voir la parution récente chez Akashic books (éditeur américain) de recueils de nouvelles centrés autour d'une ville, : D.C. Noir, Dublin Noir, Brooklyn Noir, etc. qualifiées par l'éditeur d'« original noir anthologies».

12.L'expression « film noir » aux Etats-Unis désigne une certaine production des années quarante et cinquante, avec des films comme Gilda, de Charles Vidor, The Lady from Shanghai, d'Orson Welles, ou The Night of the hunter, de Charles Laughton. Mais elle est aussi employée pour qualifier des œuvres récentes, comme celle de Quentin Tarantino. 13.Par exemple, une étude de William Marling, professeur d'anglais à l'Université de Cleveland, a pour titre The American roman noir : Hammett, Cain and Chandler (The University of Georgia Press, 1995).

14. C'est le cas dans beaucoup de romans noirs contemporains : la plupart de ceux de Thierry Jonquet, Jean-Bernard Pouy, Claude Amoz, Jean-Hugues Oppel, Dominique Manotti, Pascal Dessaint, Pascal Garnier ou Maurice G. Dantec, pour citer les auteurs les plus connus. 
15.Richard Saint-Gelais, "Rudiments de lecture policière ", in Revue belge de philologie et

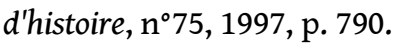

16. Gérard Genette, Figures III, Seuil, p.78.

17. Les « anachronies narratives » sont « les différentes formes de discordance entre l'ordre de l'histoire et celui du récit ». Gérard Genette, Figures III, op. cit. p 79.

18. Alain Rabatel, « La dialogisation au cœur du couple polyphonie/ dialogisme chez Bakhtine », in Revue Romane ${ }^{\circ} 41,1$, avril 2006, p. 56-57.

19. Voici la définition du « collage » dans le DITL : « Le plus souvent, et dans le sens le plus proche de celui qui s'est imposé pour la peinture, le terme désigne l'enchâssement, dans un texte en prose ou en vers, et par le biais d'une transposition graphique, d'éléments empruntés à des discours non littéraires, préexistants, comme, également des coupures de journaux, des slogans publicitaires, des affiches ou extraits d'affiches, des cartes de visite, des chansons populaires, etc. » DITL, en ligne sur http ://www.ditl.info/ arttest/art1213.php, [consulté le 27/01/06].

20. C'est le propre de la poétique conditionaliste, « dans des jugements où le diagnostic de littérarité équivaut à un label de qualité : comme lorsqu'un partisan du critère fictionnel refuse néanmoins de l'accorder à un roman de quai de gare, le jugeant trop "mal écrit" pour "être de la littérature" - ce qui revient en somme à considérer la fictionalité comme une condition nécessaire mais non suffisante de la littérarité ». Gérard Genette, Fiction et diction, Seuil, 1991, p. 27.

21. Michal Glowinski, «Sur le roman à la première personne ", Poétique $n^{\circ} 72$, novembre 1987, p. 500.

22. Ibid.

23. Ibid.

24. Genette oppose la " poétique essentialiste », qui tient pour « acquise, définitive et universellement perceptible la littérarité de certains textes » et la « poétique conditionaliste » qui pose la question de savoir « à quelles conditions, ou dans quelles circonstances, un texte peut-il, sans modifications internes, devenir une œuvre ?». Fiction et diction, op. cit., p. 14.

25. J. L. Borges, « Le roman policier », in Conférences, Gallimard, 1985, p. 189.

26. Jean-Louis Dufays, Stéréotype et lecture, op. cit. p. 123.

27. Richard Saint-Gelais, « Rudiments de lecture policière », art. cit. p. 790.

28. Pour Genette, l'architextualité est la relation générique entre les textes. Palimpsestes, Seuil, 1982, p. 12.

29. Ibid.

30. Par exemple, cette quatrième de couverture qui se termine ainsi : «Si le lecteur se doute qu'il existe un lien entre la quête et l'enquête, bien malin celui qui trouvera avant la fin une solution à double tranchant comme seule la vie sait en construire. » Terminus Plage, d'Alain Wagner, Actes Sud, 2005 (Babel Noir).

31. Wolfgang Iser, L'Acte de lecture, Mardaga, 1985, p. 319.

32. Denis Mellier, « Double policier et Trilogie new-yorkaise : Paul Auster et la littérature policière », in Formes policières du roman contemporain, La Licorne, n44, 1998, p. 199.

33. Wolfgang Iser, L'Acte de lecture, op. cit., p. 380.

34. La « tension narrative » est un effet littéraire qui consiste à « retarder l'exposition d'un élément du discours que le lecteur est conduit à attendre ou à anticiper avec impatience ». Raphaël Baroni, « Tension narrative, curiosité et suspense : les deux niveaux de la séquence narrative », en ligne sur http ://www.vox-poetica.org, [consulté le 15/05/04]. 
35. Richard Saint-Gelais, « Rudiments de lecture policière », art. cit. p. 793. Nous soulignons.

36.Paul Ricœur, Temps et récit, t.1, Points Seuil, 1983, p. 10.

37.Stefano Tani, « Le détective écartelé », in Diogène $n^{\circ} 120,1982$, p. 28-29.

\section{RÉSUMÉS}

Aujourd'hui, les romans noirs ont en commun une intrigue liée à une transgression criminelle des règles sociales. Ce cadre thématique, qui peut donc se définir comme un stéréotype, permet de classer ces romans dans le domaine de la littérature de genre, laquelle est encore largement assimilée à la paralittérature. Pourtant, le genre noir joue constamment sur son appartenance au domaine paralittéraire en travaillant sur les stéréotypes qui le définissent. En effet, ceux-ci autorisent le renouvellement du genre par l'emploi de procédés narratifs qui construisent des romans hétérogènes, où la progression est discontinue. L'emploi de ces procédés narratifs concerne aussi bien le point de vue - multiplication des personnages focalisateurs - que l'ordre du récit, avec des retours en arrière qui entretiennent des liens fondamentaux avec la reconfiguration de l'intrigue, ou encore le collage de discours non-fictionnels. Cette hétérogénéité narrative donne toute sa place à la question de la réception, puisqu'il s'agit de contourner les attendus des stéréotypes thématiques par des structures narratives qui les disséminent, en jouant avec le lecteur.

INDEX

Mots-clés : réception, roman noir, paralittérature, stéréotype, hétérogénéité

Keywords : narration

\section{AUTEUR}

\section{ANISSA BELHADJIN}

Maître de conférences de littérature française à l'Université de Cergy-Pontoise, CRTF 